\title{
Prevalence of intestinal parasites among type I diabetic patients in pediatrics Zagazig university hospital
}

\begin{abstract}
Background: Children with diabetes mellitus (DM) are known to be at an increased risk of infections; however, little is known about the prevalence of intestinal parasitic infections among them. Objective: The aim of this study was to assess the prevalence of intestinal parasitic infections among Type 1 Diabetes Mellitus (T1DM) patients in Pediatrics Zagazig University Hospitals as well as the risk factors involved in the acquisition of these parasitic infections. Material and Methods: This cross sectional study was conducted on 185 type 1 diabetic patients from November 2017 to November 2018. Full history, general examination, random blood sugar test (RBS), $\mathrm{Hb}$ and hemoglobin A1c levels were performed for each participant. Fresh faecal samples were collected transported and examined by direct wet, iodine stained smears, formol-ether concentration and modified ziehl neelsen staining. The rates of parasitic infections were calculated and data were analyzed by SPSS software.

Results: The rate of parasitic infection was (27\%). The most detected infection in our study was Cryptosporidium followed by B. hominis, G. lamblia and H. nana, the rate of infection with protozoa especially opportunistic ones was higher than helminthic infections. A high significant association $\mathrm{P}(<0.001)$ between intestinal opportunistic parasitic infection and diabetic status regarding HbA1c, RBS and DKA. Conclusion: Patients with DM might be at an increased risk of infection with intestinal parasites specifically opportunistic infections causing regression in their anthropometric measures and HB levels and so, routine stools examination should be considered for them.
\end{abstract}

Keywords: diabetes mellitus (DM), T1DM patients, intestinal parasitic infections and diabetic status.
Volume 7 Issue 6 - 2019

\author{
Zainab Esmail El Drawany,' Safaa Hamdy \\ Ahmed Saleh,' Samia El sayed Etewa, ${ }^{2}$ \\ Shereen Mahmoud Ibrahim² \\ 'Department of Pediatrics, Faculty of Medicine, Zagazig \\ University, Egypt \\ ${ }^{2}$ Department of Medical Parasitology, Faculty of Medicine, \\ Zagazig University, Egypt
}

\begin{abstract}
Correspondence: Shereen Mahmoud Ibrahim, Department of Medical Parasitology, Faculty of Medicine, Zagazig University, Zagazig 445 I 9, Egypt, Tel (+2) 0 I 2 I 2756868,

Email shery.redberry@gmail.com,shmhmoud@zu.edu.eg
\end{abstract}

Received: August 9, 2019 | Published: November 25, 2019

\section{Introduction}

Diabetes mellitus (DM) is a metabolic non-communicable disease characterized by high blood glucose levels leading to the classical symptoms of polyuria, polyphagia and polydipsia, This elevated blood glucose is either because enough insulin is not produced by the body or because cells lack responsiveness to the insulin produced. ${ }^{1}$ It is a widely distributed disease, with a rapidly increasing prevalence in various populations. ${ }^{2}$ There are two types of diabetes mellitus: Type 1, Insulin-Dependent Diabetes Mellitus (IDDM)1and Type 2 or Non-Insulin-Dependent Diabetes Mellitus (NIDDM). ${ }^{3}$ Type-1DM is partly inherited, and then triggered by certain infections, with some evidencelpointing at Coxsackie B4 virus. A1genetic element in individuals susceptible to some of these triggers has been traced to particular human leukocyte antigen genotypes.1However, even in those who have inherited the susceptibility,1type $1 \mathrm{DM}$ seems to require an environmental trigger. ${ }^{4}$ It was reported that any weakness of immune system such as chronic internal diseases and metabolic disorders including Diabetes puts patients at higher risks of infectious diseases. ${ }^{5}$ ilntestinal parasites also constitute a major public health problem especially in the developing countries where poor sanitation and information lack result in contamination of water and food with continuance of the parasitic life cycles, 1 they are also one of the most important causes of morbidity and mortality although they usually cause non-1aggressive diseases. ${ }^{6}$

Parasitic infections are among the most widespread of all chronic human infections worldwide.1About 340 parasites infect more than 3.5billion persons and cause apparent clinical signs and symptoms with varying morbidity and mortality in 450 million approximately. ${ }^{7}$ Sabah and Temsah, ${ }^{8}$ stated that, infections with intestinal parasites are uncommon to cause high morbidity or mortality to man, but they are risky to diabetic patients. Even in communities with adequate sanitary conditions and higher educational levels, 1 some of these parasites play an important role in causing diseases especially in specific groups such aslimmunocompromised patients and younger children. ${ }^{9}$ Diabetic patients are known to be immunocompromised,1and intestinal parasites are importantlopportunistic pathogens causing infections among immunosuppressedlpatients. ${ }^{10}$ Many relationships between DM and parasitic infections were reported such as the predisposition of diabetic patients to intestinal parasitic infections reported by El Nadi et al. ${ }^{11}$ in Upper Egypt, who stated that intestinal parasites usually create benign diseases, though they may induce complications with high morbidity and mortality to the immunocompromised, including the diabetics. ${ }^{12}$

Bhattacharjee et al. ${ }^{13}$ recorded also that sedentary lifestyle and anemia caused by some parasites have the potential to increase DM risk. Other relationship such as the predisposition of Metagonimus yokogawai infected patients to develop diabetes reported by Yamada et al. ${ }^{12}$ in Japan when a diabetes mellitus patient with left hemi paresis and disorientation was hospitalized because of atypical non-hypertensive multiple intracerebral hemorrhages. His stools examination showed Metagonimus yokogawai eggs and after treatment he became normal and his diabetes mellitus markedly improved so they concluded that $\mathrm{DM}$ is a chronic sign of metagonimiasis. On the other hand, a protective 
relationship between some parasites and DM was also recorded by Mendonc et al. ${ }^{14}$ who reveled that provided there are related cases of disseminated strongyloidiasis in diabetics, the immunological screening of these patients could prevent severe and fatal outcomes of DM. Soil-Transmitted Helminthic infections were also approved to be associated with a modest improvement of insulin sensitivity, that makes this infection a protective factor for DM complications. ${ }^{15}$ The present study was conducted to assess the prevalence of intestinal parasitic infections among T1DM patients in Pediatrics Zagazig University Hospitals reflecting Sharkia governorate as well as the risk factors involved in the acquisition of these parasitic infections in these patients.

\section{Subjects and methods}

This study is a cross sectional study that was performed at Pediatricsloutpatient clinics, the diabetic unit of Zagazig University Hospital and Medical Parasitology Department, Faculty of Medicine, Zagazig University from November 2017 to November 2018 on 185 T1DM patients. The participants were T1DM patients aging from 1 to $14 y e a r s$ old without previous chronic internal disease, cancer, or any immunodeficiency diseases (except for DM) who did not currently receive any immunosuppressive drug, based on individual history taking. Exclusion criteria were consumption of antibiotics, antiparasitic drugs, during previous 2 weeks, adult diabetic1patients and T1DM patients suffering from any other chronic debilitating disease like (cancer, immunodeficiency diseases, chronic chest or kidney diseases). Children with DM were randomly selected from those with a proven history of DM type I attended the Diabetic Unit and the Diabetic Clinics in Pediatrics Zagazig University Hospital for routine checkup or for admission.

Full history, general examination, random blood sugar test (RBS), $\mathrm{Hb}$ and hemoglobin A1c levels were performed for each participant. ${ }^{16}$ Also demographic data and risk factors for parasitic infections were filled for all participants. Stools samples were obtained from children after the full explanation of the process to them. Fresh faecal samples were collected in dry labeled, plastic containers, with tight fitting covers and they were taken with caution not to be contaminated with urine. ${ }^{5}$ The samples were transported immediately to the Department of Parasitology zagazig university, where lab assessments were done. Stools samples were examined by direct wet ${ }^{17}$ and iodine stained smears $^{18}$ and formol-ether concentration method for the routine screening of ova and parasites diagnostic stages (Figures 1-10). ${ }^{19}$ Each sample was examined separately. Smears were prepared from sediments of formol-ether concentration for modified ziehl neelsen staining to detect intestinal coccidian and Cryptosporidium spp. ${ }^{19}$ Slides were examined by light microscopy at $\times 100, \times 400$ and $\times 1000$ magnifications. Stools culture on nutrient agar was also done for detection of some intestinal parasites diagnostic stages. ${ }^{20}$ Patients with T1DM were divided according to the presence or absence of intestinal parasitic diagnostic stages in their stools into two main groups

Group I: T1DM Patients positive for intestinal parasites.

Group II: T1DM Patients negative for intestinal parasites.

Furthermore, group I was divided into 2 subgroups: The first included patients with T1DM positive for opportunistic intestinal parasites and the second included patients with TD1M patients positive for non-opportunistic intestinal parasites. The rates of parasitic infections were calculated as the ratio of the number of
T1DM patients with at least one positive parasitological test to the number of total T1DM patients participated in the study. ${ }^{5}$

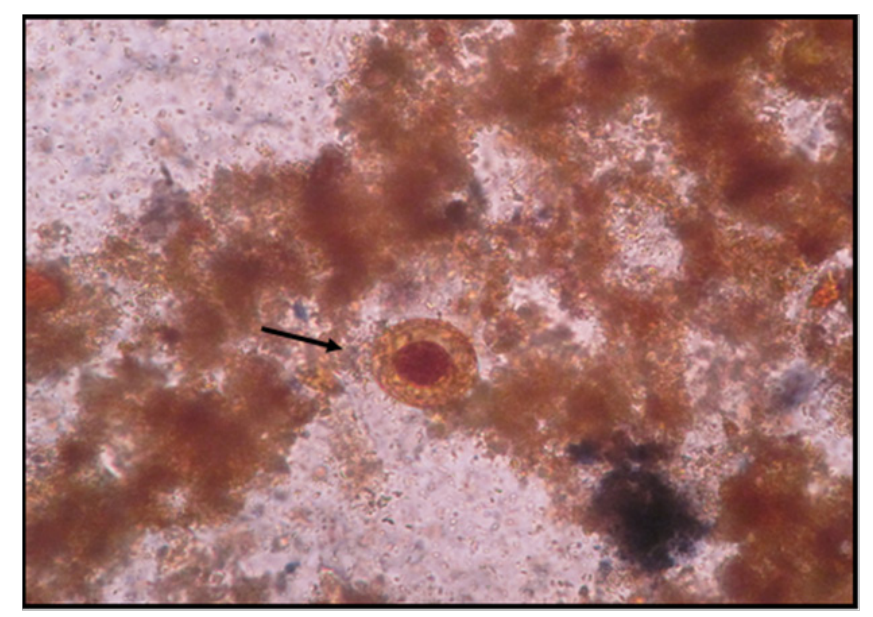

Figure I Lugol's iodine stained stools smear showing H. nana egg (x400).

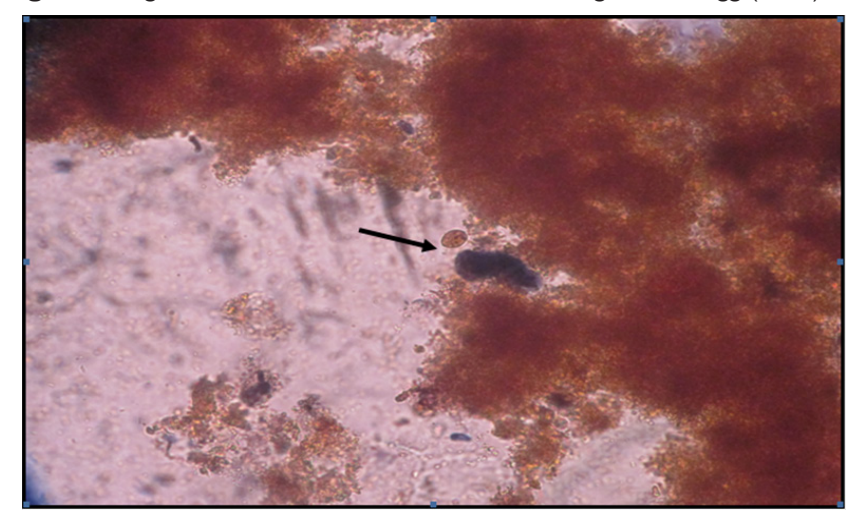

Figure 2 Lugol's iodine stained stools smear showing Giardia lamblia cyst $(\times 400)$.

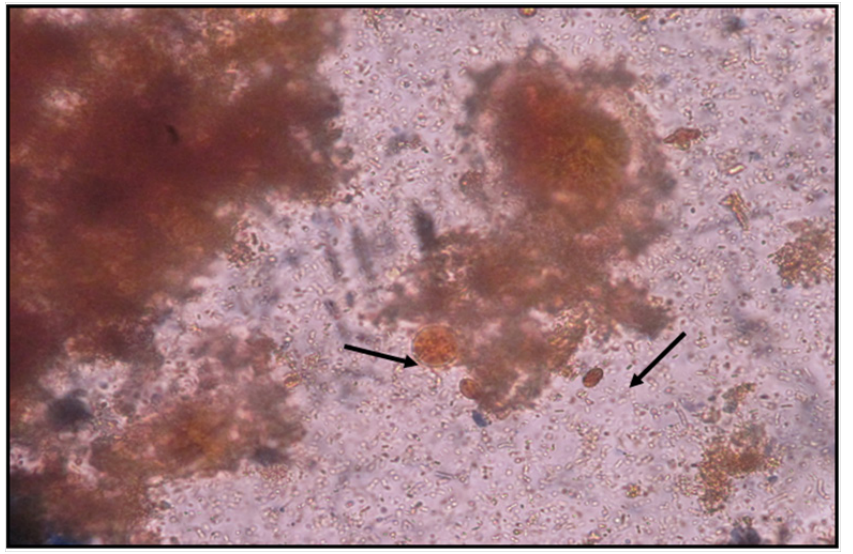

Figure 3 Lugol's iodine stained stools smear showing Giardia lamblia cyst and Entamoeba histolytica cyst (x400).

\section{Ethical consideration}

Approval was taken from the institutional review board (IRB) of faculty of medicine Zagazig University. Official permission of study setting department (Pediatrics Department of Zagazig University). Informed consents were taken from parents of all the participants. 


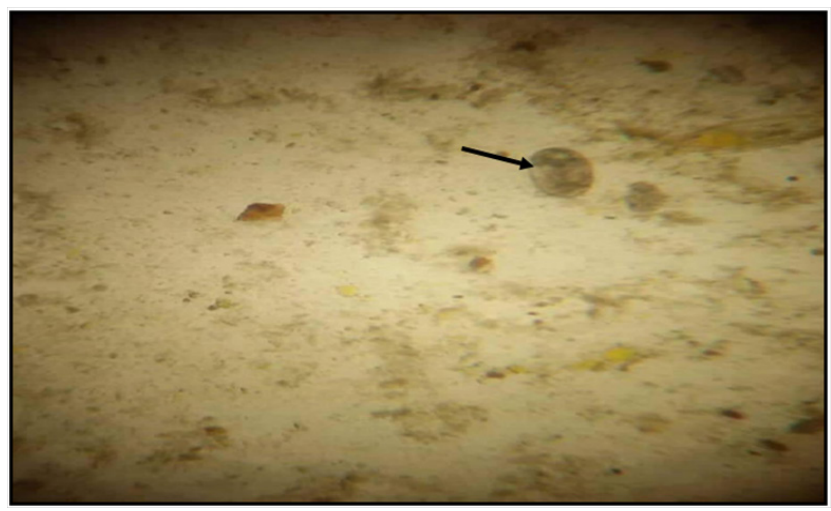

Figure 4 Lugol's iodine stained stools smear showing Giardia lamblia cyst $(x \mid 00)$.

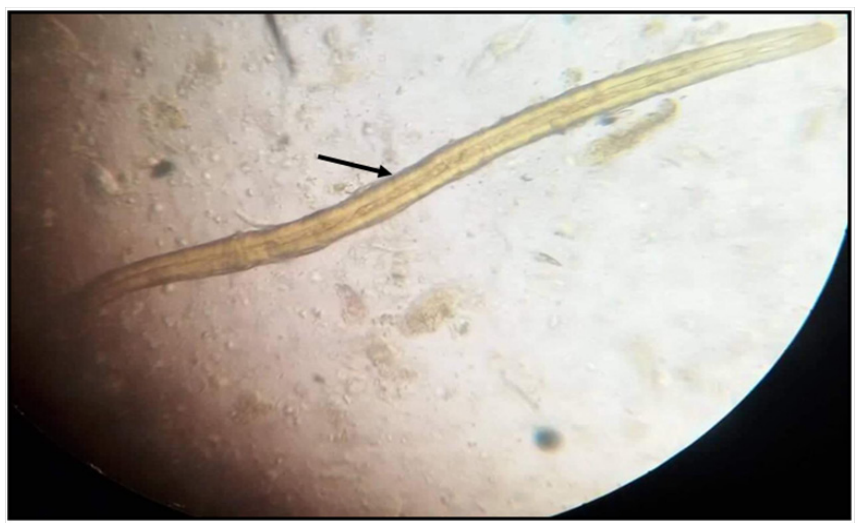

Figure 5 Lugol's iodine stained stools smear showing Strongyloids larva (x400).

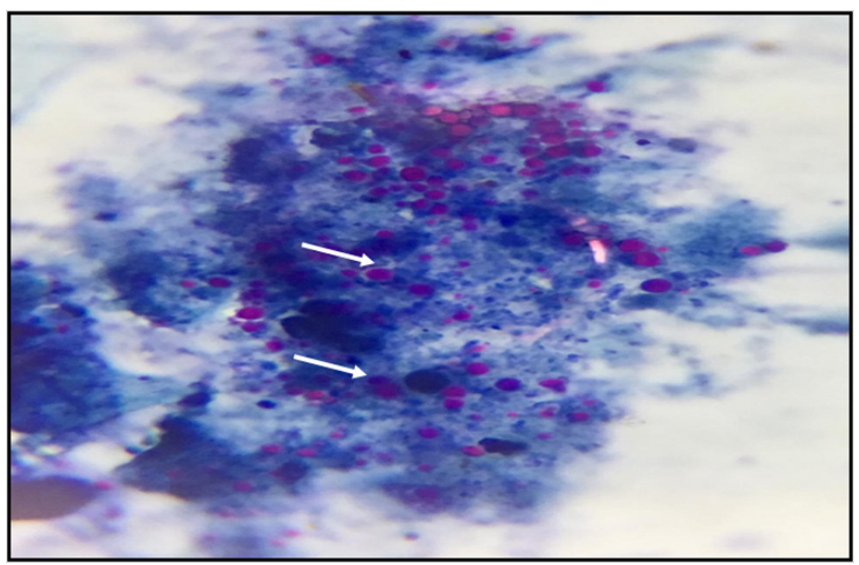

Figure 6 Stools smear stained with modified Ziehl -Neelsen stain showing Cryptosporidium spp. Oocyst (x1000).

\section{Statistical analysis}

Data were analyzed by SPSS software (version 16, SPSS Inc., Chicago, IL, USA). Rate of parasitic infection and the risk factors were compared between the two groups by Fisher's exact test where $\mathrm{P}$ $<0.005$ was considered as statistically significant.

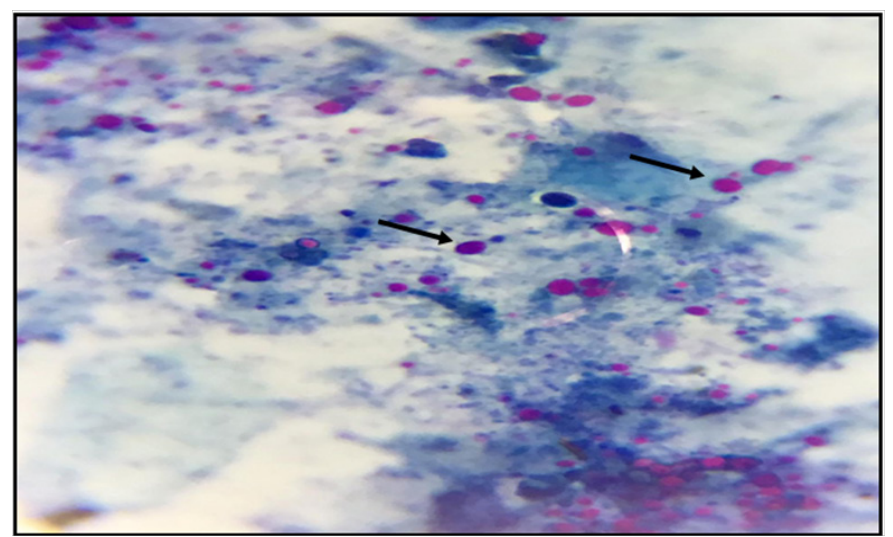

Figure 7 Stools smear stained with modified Ziehl -Neelsen stain showing Cryptosporidium spp. oocyst (xI000).

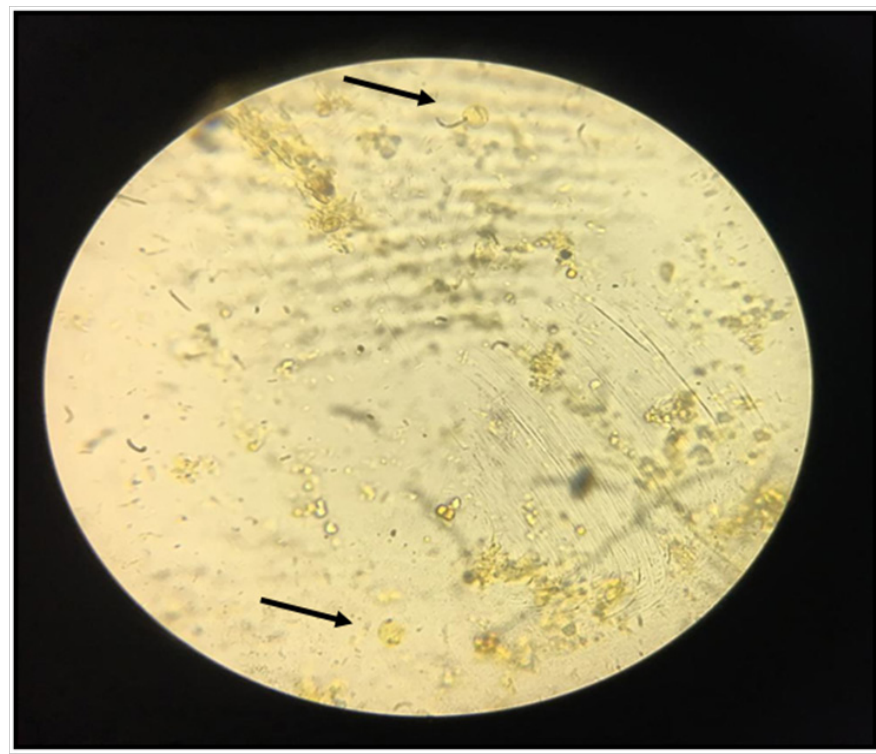

Figure 8 Lugol's iodine stained stools smear showing Blastocystis hominis cyst $(\times 400)$.

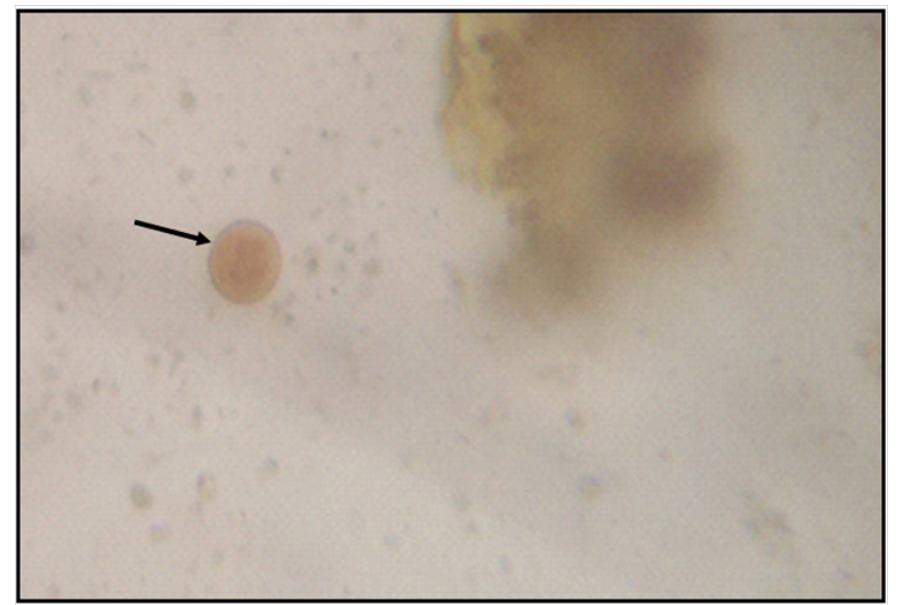

Figure 9 Lugol's iodine stained stools smear showing Entamoeba histolytica cyst (1000). 


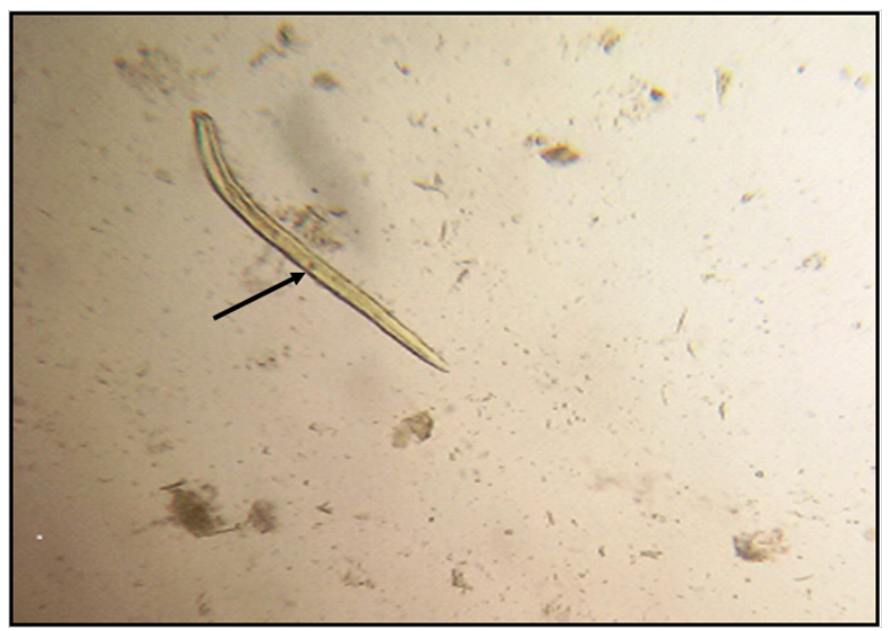

Figure 10 Lugol's iodine stained stools smear showing strongyloides larva $(x \mid 00)$.

\section{Results}

The rate of parasitic infection was (27\%) overall. From all infected children intestinal protozoal infections were higher than helminthic ones $64 \%$ and $22 \%$ respectively and opportunistic parasites showed a high percentage $(60 \%)$ among T1DM patients with intestinal parasitic infections (Table 1). And the percentages of distribution of different symptoms among the studied groups demonstrated that T1DM patients positive for intestinal parasites suffering from diarrhea alone had the highest percentage (48\%) followed by those suffering from diarrhea associated with abdominal pain and discomfort (18\%) (Table 2 ). There is a high significant difference between the two groups as regarding body mass index $\mathrm{P}(0.02)$ with lower values in the group of T1DM patients with intestinal parasitic infections. As well as Hb level with a lower mean and SD among the T1DM patients with intestinal parasitic infections rather than those negative for intestinal parasites $(9.6 \pm 2.0$ and $10.5 \pm 1.7$ respectively). Also, there is a highly significant difference $\mathrm{P}(<0.001)$ in HA1C levels being higher in T1DM patients with intestinal parasitic infections than those negative for intestinal parasites. And among T1DM patients representing with DKA, there is higher percentage of intestinal parasitic infections (78.3\%) with a high significant difference $\mathrm{P}(<0.001)$. But there is no significant difference between both groups regarding the duration of diabetes $\mathrm{P}$ (0.1) (Table 3).

The risk factor distribution among T1DM patients showed significant difference in low socioeconomic, pump using water sources, farm animal contacts and patients following dietetic regimen among T1DM patients with intestinal parasitic infections than those negative for intestinal parasites $(\mathrm{p}<0.001)$ (Table 4$)$. The ages of T1DM patients with intestinal parasitic infections ranged from 5 to 9years while those of T1DM patients negative for intestinal parasites ranged from 1 to 14years with sigzificant difference between the two groups. There is also a significant difference $\mathrm{P}(0.003)$ between the two groups regarding the residence with a higher percentage in T1DM patients with intestinal parasitic infections in rural areas $(72 \%)$. But there is no significant difference between the groups as regarding sex $\mathrm{P}$ (0.3) (Table 4).
Table I Stool analysis and culture results of the studied TIDM patients

\begin{tabular}{|c|c|c|}
\hline \multirow{2}{*}{$\begin{array}{l}\text { Variable } \\
\text { Stool analysis/culture }\end{array}$} & \multicolumn{2}{|c|}{ Studied patients $(n=\mid 85)$} \\
\hline & $N=\mid 85$ & $\%$ \\
\hline positive (Group I) & 50 & 27 \\
\hline Negative (Group II) & 135 & 73 \\
\hline Helminthes (monoinfection): & II & 22 \\
\hline H.nana & 4 & 8 \\
\hline Ascaris & 2 & 4 \\
\hline Trichuriasis & 2 & 4 \\
\hline *Strongyloids & 3 & 6 \\
\hline Protozoa (monoinfection): & 32 & 64 \\
\hline Amebiasis & 2 & 4 \\
\hline Giardiasis & 4 & 8 \\
\hline *Cryptosporidium & 16 & 32 \\
\hline Blastocystis & 10 & 20 \\
\hline Combined infections: & 7 & 14 \\
\hline Giardia + Entamoeba & 3 & 6 \\
\hline *Giardia + Cryptospora & 2 & 4 \\
\hline *Cryptosporidium + Blastocystis & 2 & 4 \\
\hline Opportunistic parasites: & 30 & 60 \\
\hline
\end{tabular}

Table 2 History and clinical presentations of intestinal parasitic infections among Group I (TDIM patient's positive for intestinal parasites)

\begin{tabular}{lll}
\hline Variables & \multicolumn{2}{c}{ Group I (n=50) } \\
& $\mathbf{N = 5 0}$ & I 00\% \\
\hline Asymptomatic & 6 & 12 \\
Diarrhea alone & 24 & 48 \\
Diarrhea+abd.Distention+abd.Pain & 9 & 18 \\
Steatorrhea & 6 & 14 \\
Mucus \& blood in stools & 5 & 10 \\
Nausea+Vomiting+abd.Pain & 0 & 0 \\
\hline
\end{tabular}

Highly significant difference in both RBS and HA1C levels $\mathrm{P}(<0.001)$ are present being higher in T1DM patients with opportunistic intestinal parasitic infections rather than other diabetic children either free or having non opportunistic intestinal parasitic infections, also a significant difference in $\mathrm{Hb}$ level with lower means in T1DM patients with opportunistic intestinal parasitic infections rather than other T1DM patients either free or having non opportunistic intestinal parasitic infections is shown in (Table 5). This table also shows that among T1DM patients representing with DKA, there is a higher percentage of opportunistic intestinal parasitic infections (78.3\%) more than both groups of T1DM patients either those positive for non opportunistic intestinal parasites $(0.0 \%)$ or those negative for intestinal parasites with a high significant difference $\mathrm{P}(<0.001)$. 
Table 3 Relationship between intestinal parasitic infections, BMI, Laboratory investigations and diabetic status in the studied TIDM patients

\begin{tabular}{lllllll}
\hline Varient & RBS & HB & HBAIC & DM Duration & DKA (23) & BMI \\
Mean \pm SD & & & & & & \\
\hline GI & $267.6 \pm 78.4$ & $9.6 \pm 2.0$ & $9.0 \pm 1.8$ & $4.1 \pm 2.0$ & $18(78.3 \%)$ & $10.2 \pm 2.4$ \\
GII & $258.4 \pm 68.8$ & $10.5 \pm 1.7$ & $8.2 \pm 2.0$ & $4.4 \pm 3.4$ & $5(21.7 \%)$ & $12.9 \pm 2.1$ \\
P & 0.6 & $0.01 *$ & $0.01 *$ & 0.1 & $<0.001 * *$ & $0.02 *$ \\
\hline
\end{tabular}

Table 4 Distribution of risk factors to intestinal parasitic infections among the studied TIDM patients

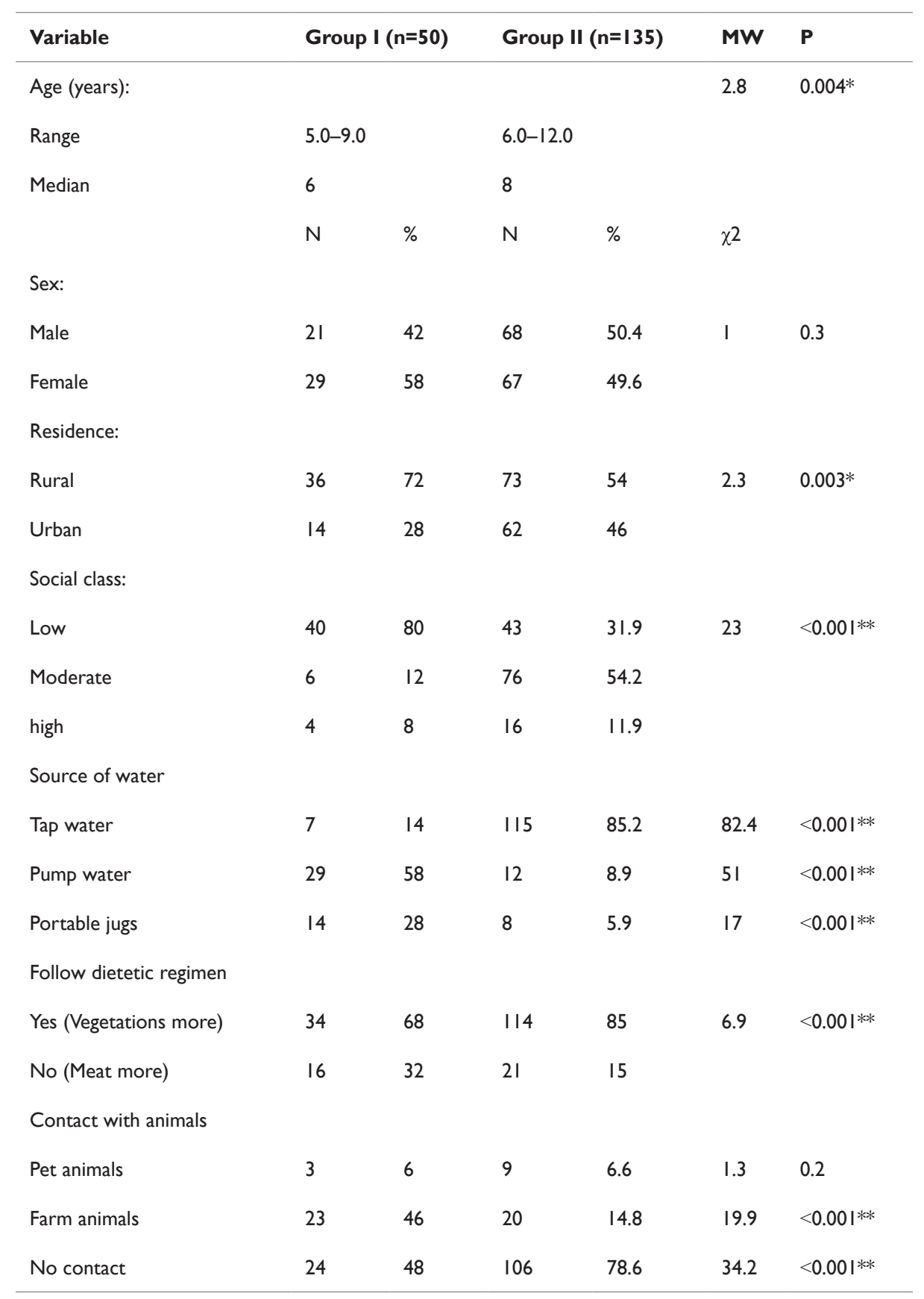


Table 5 Relationship between opportunistic intestinal parasitic infections and Laboratory investigations in the studied TIDM patients

\begin{tabular}{|c|c|c|c|c|c|}
\hline Variables & $\begin{array}{l}\text { Opportunistic } \\
\text { intestinal parasites } \\
(n=30)\end{array}$ & $\begin{array}{l}\text { Non- opportunistic } \\
\text { intestinal parasites } \\
(n=20)\end{array}$ & $\begin{array}{l}\text { Group II } \\
(n=135)\end{array}$ & f & $\mathbf{P}$ \\
\hline \multicolumn{6}{|l|}{$\mathrm{Hb}:(\mathrm{g} / \mathrm{dl})$} \\
\hline Mean $\pm S D$ & $9.5 \pm 1.2$ & $10.2 \pm 1.5$ & $10.5 \pm 1.7$ & 4.8 & $<0.00$ I $^{* *}$ \\
\hline \multicolumn{6}{|l|}{ RBS } \\
\hline Mean \pm SD & $* 300.3 \pm 94.3$ & $230.3 \pm 42.9$ & $258.4 \pm 68.8$ & 6.5 & $<0.00$ I** \\
\hline \multicolumn{6}{|l|}{ HAIC } \\
\hline Mean $\pm S D$ & $* 10.3 \pm 1.9$ & $8.0 \pm 1.1$ & $8.2 \pm 2.0$ & 15.7 & $<0.00$ I** \\
\hline DKA: & & & & $\chi^{2}$ & \\
\hline Positive & *I8 (78.3\%) & $0(0.0 \%)$ & 5 (21.7\%) & 73 & $<0.00$ I** \\
\hline Negative & 7 (4.3\%) & 25 (I5.4\%) & I 30 (80.3\%) & & \\
\hline
\end{tabular}

\section{Discussion}

For the best of our knowledge, little is known about the prevalence of intestinal parasitic infections among T1DM patients in Egypt generally and in our locality specially and against this background the present study is the first one to measure this. In this study, Intestinal parasitosis among T1DM patients was diagnosed in 50 out of 185 T1DM patients $(27 \%)$ this is higher than the prevalence previously recorded by Akinbo et al., ${ }^{2}(18.7 \%)$. And lower than that reported by Nazligul et al., ${ }^{10}(47 \%)$. These changes may be attributed to geographical location that may account for these differences as these studies were conducted in Nigeria and Anatolia whereas our study was carried out in Egypt and was implicated on T1DM patients while others included all diabetic cases. The most detectable parasitic infection in our study was Cryptosporidium followed by B. hominis, G. lamblia and H. nana, the rate of infection with protozoa especially opportunistic ones was higher than helminthic infections. In our work the rate of Cryptosporidium infection was the highest which was in accordance with a similar study by Akhlaghi et al..$^{21}$ in which Cryptosporidium infection was meaningfully more in patients with DM. In this study, Blastocystis was the second prominent intestinal parasitic infection among T1DM patients. This finding was totally supported by Mohtashamipour et al. ${ }^{5}$ findings. Mucosal invasion and intestinal inflammation have been shown in animal models of blastocystosis, ${ }^{22}$ the theory of impaired intestinal mucosal integrity in DM might explain the increased rate of Blastocystis infection in our T1DM patients that resulted in more GIT complaints.

Helminthes were the least prevalent intestinal parasites in this study. This finding was in accordance with other many studies on diabetic patients ${ }^{23}$ and. $^{2}$ In our locality, this is mostely due to the massive administration of anti-helminthic drugs by our government (deworming campaigns) which recently helped to eradicate intestinal helminthes in school children but intestinal protozoa still pose a threat. In this study, S. stercoralis was found in 3 cases and this was supported by the findings of Mendonc et al. ${ }^{14}$ in Brazil who revealed a positive association between S. stercoralis infection and DM. The most common presentation among our studied TD1M patients positive for intestinal parasites was diarrhea $(74.0 \%)$ followed by diarrhea associated with both abdominal Pain, and discomfort (44.0\%). This was in accordance with Mohtashamipour et al. ${ }^{5}$ who found that the rate of intestinal parasitic infection was more in patients with symptoms such as diarrhea, abdominal Pain, and abdominal discomfort.

However, Asymptomatic TD1M patients positive for intestinal parasites were more in a study to Tangi et al., ${ }^{24}$ but this may be explained by the great variation in age groups between his study and ours. Regarding body mass index (BMI), there was a significant difference $\mathrm{P}(0.02)$ between the TD1M patients positive for intestinal parasites and those negative for intestinal parasites in the current study. This was in accordance with Yones et al. ${ }^{25}$ Who recorded that intestinal parasitic infection have been linked with an increased risk for growth deficits in children. It is also known that high ascarisis intensity and some other parasitic infections influence the nutritional status in children and the most important parasites related to malnutrition are intestinal parasites. ${ }^{26}$ Regarding the haemoglobin level in our studied children there was a significant difference $\mathrm{P}(0.01)$ between TD1M patients positive for intestinal parasites and those negative for intestinal parasites. This was explained by Agbolade ${ }^{27}$ that parasitic infections are known to cause anemia, Diminished erythropoietin production has been also reported as the cause of anemia in DM patients. ${ }^{28}$

Moreover, helminth infections are known causes of anaemia and protein-energy malnutrition and approximately cause half of the child mortality in developing countries. ${ }^{29}$ The duration of diabetes in our studied cases had no significant difference $\mathrm{P}(<0.001)$ between TD1M patients positive for intestinal parasites and those negative for intestinal parasites T1DM patients. This was in accordance with ${ }^{2}$ who recorded the absence of any association between intestinal parasitic infections and the type or the duration of DM. In the present work, a significant difference $\mathrm{P}(0.01)$ between both groups regarding the HbA1C was noticed. Many studies approved that, for trematode infections, positive association with HbA1c concentrations were 
reported from several studies ${ }^{30}$ and. ${ }^{31}$ On the conterary, Htun et al. ${ }^{26}$ recorded no significant association of $\mathrm{HbAlc}$ with any intestinal parasitic infectious agents, while this was observed in non T1DM patients and even their regression analysis pointed to statistically nonsignificant increases in $\mathrm{HbA1c}$ concentrations at the 6-month followup after anti parasitic treatment in those patients.

In this study being uncontrolled diabetic (HA1C $>7.5$ or admitted with DKA) was associated with higher rate of intestinal parasitic infections especially opportunistic ones $(72.0 \%)$ than those with controlled diabetes. The rate of Cryptosporidium, Blastocystis and Strongyloids infections were higher in those patients, this is partially agreed with astudy of Akhlaghi, ${ }^{21}$ in which only Cryptosporidium infection was meaningfully more in his DM patients. These results indicate that the clearance of parasites from intestine might have been impaired in T1DM patients, this can be explained that both innate and acquired immunities are impaired in T1DM patients and that the functions of neutrophil such as phagocytosis and chemotaxis are impaired with a general immunosuppressive condition. ${ }^{32}$ Also impaired mucosal integrity, due to defective microcirculation in T1DM patients can predispose to intestinal parasitic infections. ${ }^{33}$

On the contrary, The rate of Cryptosporidium infection was lower in the setting of a study of Mohtashamipour et al., ${ }^{5}$ but other opportunistic parasites like Blastocystis was high and this can be explained by the clean source of water and environmental sanitation in their study area. In this study, the ages of TD1M patients positive for intestinal parasites ranged from 5 to 9years with high statistically significant results. Mohtashamipour et al. ${ }^{5}$ recorded also that, parasitic infection was more prevalent in persons under 10years old and DM patients, they reported that the reason for this was unclear as DM patients were asymptomatic, had controlled glucose level and were not immunocompromised. We can add that the children in this age are prone to parasitic infections more than elder ones. Our investigations reveals that there are more female participants $(51.9 \%)$ who were also more infected $(58 \%)$ with intestinal parasites than males, this is similar to the study in Sanliurfa province ${ }^{10}$ and another one by Mohtashamipour et al..$^{5}$ in Iran. And this was explained by Tangi et al. ${ }^{24}$ that there is a fact of a high prevalence of diabetes mellitus occurring in females than in males. In the present work, there were more parasitic infections in T1DM patients inhabiting rural areas $(62 \%)$ this was explained by de Cassia et al, ${ }^{34}$ that individuals living primarily in rural areas of low-income countries commonly harbor multiple parasitic infections, including infection with multiple helminth species.

Somphou et al., ${ }^{35}$ noted also that, children living in the rural districts have high prevalence of helminth infections and malnutrition. Helminth infections and multiparasitism are significantly associated with observed morbidities. On the other hand our results were against a study done by Tangi et al. ${ }^{24}$ who stated that there were more parasitic infections in the urban areas and explained that due to migration of diabetic patients from the rural to urban areas for more medical care in the cities hospitals. In our study, Intestinal parasitic infections were more prevalent among patients of low and moderate socioeconomic standards with a high significant value $\mathrm{P}(<0.001)$. This was mainly explained by the presence of many risk factors among those patients such as the type and source of food, the source of water, the environmental sanitation and contact with animals.

On the contrary, Tangi et al. ${ }^{24}$ mentioned in their study that since most diabetic patients are old, intestinal parasites were even more prevalent among business people and civil servants probably due to their busy schedules which make it difficult for them to visit the doctor regularly. Also they spend most of their time out of their homes hence possibly feeding out of the house from places where sanitary conditions are questionable. In our study there was significant difference $\mathrm{P}(<0.001)$ between TD1M patients positive for intestinal parasites and those negative for intestinal parasites T1DM patients regarding the type of food commenly consumed with more infection among vegetable consumers than meat consumers (68\% and $32 \%)$ respectively. The vegetables and fruits are mostly bought by diabetic patients especially after fasting to control blood sugar as they are advised to eat this type of food which is bought through handlers who sell fruits and food by the road side and this increases the risk of infection with Entamoeba histolytica cysts. ${ }^{24}$ The source of water also playes a role in intestinal parasitic infections, it was recorded that Cryptosporedium parvum causes $50.8 \%$ of water-borne diseases, and in our developing countries, $8-19 \%$ of diarrheal diseases can be attributed to Cryptosporidium spp. ${ }^{36}$

On the other hand, Mohtashamipour et al. ${ }^{5}$ stated that, the level of education, occupation and source of water were not significantly associated with intestinal parasitic infections among their diabetic cases in Iran. This may be attributed to the different age group and geographic locations of the studied patients. Regarding animal contact, there was a high significant difference towards farm animals contact among the parasite positive group $(\mathrm{P}<0.001)$. This was in accordance to Mohtashamipour et al. ${ }^{5}$ who recorded a higher rate of infection (53.8\%) among those who kept animals at home. The present work revealed a high significant association $\mathrm{P}(<0.001)$ between intestinal opportunistic parasitic infection and diabetic status regarding HbA1c, RBS and DKA. In a previous study of Tangi et al. ${ }^{24}$ They emphasized that the presence of Cryptosporidium only in the diabetic patients reinforced the theory of diabetics being immunologically weaker. Baiomy et al..$^{23}$ in Greater Cairo, studied the prevalence of the opportunistic parasites among immunocompromised patients of whom 30 were diabetic selected from Al Azhar University Hospitals , their results showed that the opportunistic parasites were in $6 \%$ of those diabetic patients. Also In a study of Mendonc et al. ${ }^{14}$ in Brazil a positive association was found between S. stercoralis infection and DM with a high mortality risk among poorly controlled DM patients. In the present work, Blastocystis hominis parasite was revealed in many cases with uncontrolled Diabetes and this was in consistence with Marcos and Gotuzzo ${ }^{37}$ who also stated that while there have been many doubts about pathogenic role of Blastocystis in humans, now it is accepted as a potential pathogen which can specifically trouble immunocompromised hosts.

Finally, being a T1DM patient is a risk factor for acquiring intestinal parasitic infection especially if uncontrolled and that makes the regular screening of T1DM patients for intestinal parasitosis is a must in our locality hospitals. On the contrary, Tangi et al..$^{24}$ approved that being diabetic was not a risk factor for acquiring intestinal parasitic infection but instead decreases the patient, $\mathrm{s}$ chances of acquiring the parasites; this may be attributed to the variations of the environmental sanitation measures and services. Also diabetic patients there visit the doctor regularly, consult, follow the doctor's order diligently and are well taken care of by their family members.

\section{Conclusion}

Patients with DM might be at an increased risk of infection with intestinal parasites specifically opportunistic infections causing 
regression in their anthropometric measures and $\mathrm{HB}$ levels and so, routine stools examination should be considered for them.

\section{Acknowledgments}

Authors gratefully acknowledge Nurses of Endocrine and General Unit for their valuable cooperation in sample collection.

\section{Conflicts of interest}

Authors have no conflict of interest.

\section{Funding}

None.

\section{References}

1. David G, Gardner T, Dolores R. Greenspan's basic and clinical endocrinology. $9^{\text {th }}$ Edition. New York: McGraw-Hill Medical. 2011;200-220.

2. Akinbo F, Olujobi O, Omoregie R, et al. Intestinal parasitic infections among diabetes mellitus patients. Biomarkers and Genomic Medicine. 2013;5(1-2):44-47.

3. Belmokhtar F, Belmokhtar R, Dali-Sahi M. Risk factors associated with type 2 diabetes mellitus in west region of Algeria. Maghnia J Diabetes Metab. 2011;2:148-150.

4. Rise'rus U, Willett WC, Hu FB. Dietary fats and prevention of type 2 diabetes. Progin Lipid Res. 2009;48(1):44-51.

5. Mohtashamipour M, Ghaffari Hoseini Sh, Pestehchian N, et al. Intestinal parasitic infections in patients with Diabetes Mellitus: A case-control study. J Anal Res Clin Med. 2015;3(3):157-163.

6. Gil F F, Barros MJ, Macedo NA. Prevalence of intestinal parasitism and associated symptomatology among hemodialysis patients. Rev Inst Med Trop Sao Paulo. 2013;55(2):69-74.

7. WHO. Infectious Diseases Home Page. Intestinal Parasites. 2014.

8. Sabah AA, Temsah AG. Prevalence of some gastro-intestinal parasites in diabetic patients in Tana City, gharbia governorate, Egypt. $J$ Egypt Soc Parasitol. 2015;45(3):681-684.

9. Fantry L. Gastrointestinal infections in the immuno-compromised host. Curr Opin Gastroenterol. 2002;18(1):34-39.

10. Nazligul Y, Sabuncu T, Ozbilge H. Is there a predisposition intestinal parasitosisin diabetic patients? Diabetes Care. 2001;24(8):1503-1504.

11. El Nadi NA, Hassanien HA, Ahmad AM, et al. Intestinal parasites in diabetic patients in Sohag University Hospitals, Egypt. J Egypt Soc Parasitol. 2015;45(2):443-449.

12. Yamada SM, Yamada S, Takada H, et al. A case of metagonimiasis compli- cated with multiple intracerebral hemorrhages and diabetes mellitus. J Nippon Med Sch. 2008;75(1):32-35.

13. Bhattacharjee S, Kalbfuss N, Prazeres da Costa C. Parasites, microbiota and metabolic disease. Parasite immunology. 2017;39(5).

14. Mendonca SCL, Goncalves-Pires MdRF, Rodrigues RM, et al. Is there an association between positive Strongyloides stercoralis serology and diabetes mellitus? Acta Tropica. 2006;99(1):102-105.

15. Wiria AE, Hamid F, Wammes LJ, et al. Infection with Soil-Transmitted Helminths Is Associated with Increased Insulin Sensitivity. PloS one. 2015;10(6):0127746.
16. Lorenzo C, Wagenknecht LE, Hanley AJ, et al. A1C between 5.7 and $6.4 \%$ as a marker for identifying pre-diabetes, insulin sensitivity and secretion, and cardiovascular risk factors: the Insulin Resistance Atherosclerosis Study (IRAS). Diabetes Care. 2010;33(9):2104-2109.

17. Fleck SL, Moody AH. Diagnostic technique in Medical Parasitology. $1^{\text {st }}$ Edition. Butterworth\&Co. (publishers) Ltd. 1988;22-26.

18. Cheesbrough M. Medical Laboratory Manual for Tropical Countries. ELBS Tropical Health Technology Butterworth. 1987;561-564.

19. Chessbrough M. District laboratory practice in tropical countries. Part I, Cambridge University press. Low Pric Editions. 2007;196- 207.

20. Arakaki T, Masaaki I, Fukunori K, et al. Efficacy of Agar-plate Culture in Detection of Strongyloides stercoralis Infection. J Parasitol. 1990;76(3): 425-428.

21. Akhlaghi L, Gharavi M, Faghihi A, et al. Survey on the prevalence rates of intestinal parasites in diabetic patients in Karaj and Savodjbolagh cities. Razi j Med Sci. 2005;12(45):23-29.

22. Abdel-Hameed DM and Hassanin OM. Proteaese activity of Blastocystis hominis subtype 3 in symptomatic and asymptomatic patients. Parasitol Res. 2011;109(2): 321-327.

23. Baiomy AMS, Mohamed KA, Ghannam MA, et al. Opportunistic parasitic infections among immuno-compromised Egyptian patients. J Egypt Soc Parasitol. 2010;40(3):797-808.

24. Tangi FB, Fokam EB, Longdoh NA. Intestinal parasites in diabetes mellitus patients in the Limbe and Buea municipalities, Cameroon. Diabetes Res Open J. 2016;2(1):147-153.

25. Yones A, Lamia A, Alameldin M, et al. Effect of enteric parasitic infection on serum trace elements and nutritional status in upper Egyptian children. Departments of Medical Parasitology and Pediatrics, Faculty of Medicine, Assiut University, Assiut, Egypt. 2015;5(1):11-17.

26. Htun NSN, Peter O, Ivan M, et al. Association between gastrointestinal tract infections and glycated hemoglobin in school children of poor neighborhoods in Port Elizabeth, South Africa. PLOS Neglected Tropical Diseases. 2018;12(3).

27. Agbolade OM, Abimbola WA, Bolarinwa OI. Parasitic infestations, anaemia and blood glucose level in out-patients of a secondary health centre in south-western Nigeria. World J Med Sci. 2009;4:147-150.

28. Dikow R, Schwenger V, Schomig M. How should we manage anaemia with diabetes? Nephrol Dial Transplant. 2002;17(supp 1):67-72.

29. Oninla SO, Onayade AA, Owa JA. Impact of intestinal helminthiases on the nutritional status of primary- school children in Osun state, southwestern Nigeria. Ann Trop Med Parasitol. 2010;104(7):583-594.

30. Geach T. Diabetes: Helminths improve insulin sensitivity and enhance M2 macrophage numbers in WAT of obese mice. Nature reviews Endocrinology. 2015;11(6):316.

31. Chen Y, Lu J, Huang Y, et al. Association of Previous Schistosome Infection With Diabetes and Metabolic Syndrome: A Cross-Sectional Study in Rural China. The Journal of Clinical Endocrinology \& Metabolism. 2012;98(2):E283-E7.

32. Tanaka Y. Immunosuppressive mechanisms in diabetes mellitus. Nihon Rinsho. 2008;66(12):2233-2237.

33. Stark D, Barratt JLN, Van Hal S, et al. Clinical significance of enteric protozoa in the immunosuppressed human population. Clin Microbiol Rev. 2009;22(4):634-650.

34. De Cassia Ribeiro Silva R, Barreto ML, Assis AM, et al. The relative 
influence of polyparasitism, environment, and host factors on schistosome infection. Am J Trop Med Hyg. 2007;77(4):672-675.

35. Somphou S, Jürg U, Kongsap A, et al. Multiparasitism and intensity of helminth infections in relation to symptoms and nutritional status among children: A cross-sectional study in southern Lao People's Democratic Republic. Acta Tropica. 2015;141(B):322-331.
36. Gatei W, Wamae CN, Mbae C, et al. Cryptosporidiosis: prevalence, genotype analysis, and symptoms associated with infections in children in Kenya. Am J Trop Med Hyg. 2006;75(1):78-82.

37. Marcos LA, Gotuzzo E. Intestinal protozoan infections in the immunocompromised host. Curr Opin Infect Dis. 2013;26(4):295-301. 